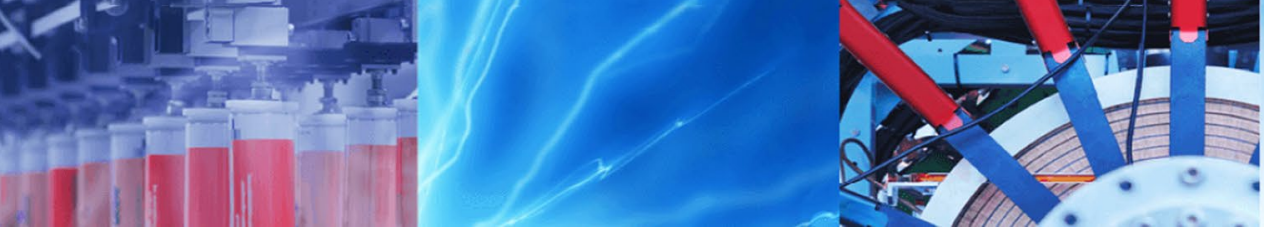

Research Article

\title{
Design and performance evaluation of Improved DFACO protocol based on dynamic clustering in VANETs
}

\author{
Sahar Ebadinezhad ${ }^{1,2}$ (1)
}

Received: 9 October 2020 / Accepted: 10 March 2021 / Published online: 20 March 2021

(C) The Author(s) 2021 OPEN

\begin{abstract}
This study focuses on Vehicular Ad-hoc Networks (VANETs) stability in an environment that is dynamic which often leads to major challenges in VANETs, such as dynamic topology changes, shortest routing paths and also scalability. One of the best solutions for such challenges is clustering. In this study, we present five novel routing protocols based on Dynamic Flying Ant Colony Optimization (DFACO) algorithm to achieve minimum number of clusters, high accuracy, minimum time and solution cost by selecting the best cluster-head which is obtained from a new mechanism of dynamic metaheuristic-based clustering. In this regard, major improvements are applied on classical DFACO by adjusting the procedure for updating the pheromone and tuning the evaporation rate that has a major role in DFACO. In this research two individual phases of experiments are conducted for performance evaluation of proposed routing protocols. The presented solution is verified and compared to classic Ant Colony Optimization (ACO), DFACO and ACO Based Clustering Algorithm for VANET (CACONET) algorithms in phase one; and compared to clustering algorithms such as Center Position and Mobility CPM), Highest-Degree algorithm (HD), Angle-based Clustering Algorithm (ACA) in phase two through NS-2 and SUMO simulation tools. Simulation results have confirmed the expected behaviour and show that our proposed protocols achieve better node connectivity and cluster stability than the former.
\end{abstract}

Keywords Vehicular Ad-hoc Networks · Clustering approach · Quality of service · Routing protocols · Dynamic flying $\mathrm{ACO}$

\section{Introduction}

A well-known combinatorial optimization problem is raised as the Vehicle Routing Problem (VRP) in transportation dialectics. The main objective of VRP is decreasing the cost of routes (such as time, distance, etc.) which are discovered in the progress of vehicular route discovery. The VANET network communication includes Vehicle-toInfrastructure (V2I) and Vehicle-to-Vehicle (V2V) which are a dynamic network, being that there are nodes having inconsistent/random motion leading to nodes frequently experiencing structural deviations. Eventually, this leads to network expiration resulting from the network separation. In order to increase its lifetime, the mobility pattern or forecasting pattern can be anticipated, which will result in the extensive usage of application in multimedia, commercial, emergency, safety and managing of traffic applications. Moreover, for data to be transmitted efficiently, it is mandatory for efficient Quality of Service (QoS). Therefore, designing an appropriate routing protocol for vehicles in large-scale is a challenging issue. Lack of network scalability is a problem that leads to a lot of damage in the network sustainability. Also, in cases of surveillance and safety application, delay can be dangerous. In this regard,

$\triangle$ Sahar Ebadinezhad, Sahar.ebadinezhad@neu.edu.tr| ${ }^{1}$ Department of Computer Information Systems, Near East University, 99138 Nicosia TRNC, Northern Cyprus. ${ }^{2}$ Computer Information Systems Research and Technology Center (CISRTC), Near East University, 99138 Nicosia TRNC, Northern Cyprus. 
optimizing vehicular networks for equal distribution of network load and scalability can be created by intelligent clustering algorithms that contribute key aspects. In this regard, five new algorithms are proposed in this study for the VANET environment with the aim of discovering a best route. On the other hand, most of the studies on the clustering field are concentrated on the static/traditional clustering method that forms the clusters based on the closeness of vehicles to the Base Station/Road Side Unit (BS/RSU) to be elected as Cluster Head (CH).

In contrast, dynamic clustering is proposed in this research which eliminates cluster formation based on RSU and considers the other metrics vehicular speed, direction and mobility pattern to form a cluster. Therefore, in this work, a unique dynamic metaheuristic-based clustering is proposed to create better dissemination and aggregation of information among vehicles.

The rest of this work is arranged as follows. In Sect. 2 there will be a brief explanation of modified ACO algorithms in recent decades and existing challenges in VANET. Section 3 depicts our proposed methodologies step by step. In Sect. 4 there will be an illustration of the simulation setup and outcomes. Section 5 discusses the experiment outcome of this research and shows the effect of proposed protocol on the network stability. The final section includes the conclusion and suggestions for future work on this topic.

\section{Related work}

\subsection{Variation of improved ACO algorithm to avoid the problem of local optimum}

Modern approaches for solving problems that have been unsolvable are introduced in everyday technology. An efficient and a trustworthy technique to overcome optimization problems are achieved by adopting evolutionary algorithms. These algorithms achieve multiple solutions through development synchronously. In this context, several metaheuristic protocols are proposed such as Ant Colony Optimization (ACO), Genetic Algorithm (GA), Artificial Bee Colony (ABC), Simulated Annealing (SA) and etc. The meta-heuristic algorithm can be enhanced via objective function and transition rule which are indispensable elements and lead to best path discovery [1]. These algorithms operate based on obtaining enhancement after each iteration. The other variation of nature-inspired metaheuristics algorithm are $A C O$ algorithm being one of the solutions [2], mine blast algorithm (MBA) [3], evaporation rate water cycle algorithm (ER-WCA) [4], salp swarm algorithm (SSA) [5], modified adaptive differential evolution (MADE) [6]. Improvement of global optimal and effective path discovery is achieved by Xu et al. [7] with introducing chaotic signals that added to dynamic movement of ants.

Consequently, to solve complex optimization problems which are multi-objective, ACO algorithms are one of the solutions. The main issues with the ACO algorithm occur by random probability selection. These drawbacks are usual stagnation, slow speed in terms of convergence, the longer time for searches, etc. On the bright side, the ACO algorithm has easy computer realization, better solution and robust abilities of discovery. Accordingly, numerous algorithms have been introduced that are improved for ACO with ambitions to vanquish the algorithm's deficiency such as gradual convergence speed, become trapped into local optimum and indistinct convergence time.

Introducing a new technique for exploration and a new scheme to evaluate pheromone value are proposed by Aljanaby et al. [8] on the basis of multiple ant colonies interaction. Selecting the next node in this research is applied based on the median of all multiple colony's pheromone values for those visited paths. Stagnation problems is the other drawback of ACO algorithm which is minimized via upgrading heuristic parameters [9]. In this study data structure technique is used to sort out the cities that are visited with minimum route distance. Moreover, entropy (heuristic information) is utilized to reduce stagnation problems. The other recent algorithm is Flying Ant Colony Optimization (FACO) which works with a combination of walking and flying ants' functions. This algorithm aimed for preserving the equilibrium of exploitation and exploration [10]. Figure 1 illustrates the process of the Dynamic Flying Ant Colony Optimization DFACO [11] algorithm which is modified from FACO and is added to the process of ACO. More detail and information about variation of the modified ACO algorithm can be found in our previous work [12].

\subsection{VANET routing protocols based on Ant colony optimization}

The proposition of VRP is introduced through truck dispatching problems by Ramser and Dantzig [13].

$E=\left\{\left(v_{i}, v_{j}\right) \in v^{2}|| x_{i}-x_{j} \mid \leq \min \left(r_{i}, r_{j}\right)\right\}$

VANET's routing protocols are an important research area. There are various routing protocols which come from MANET study like Destination-Sequenced DistanceVector (DSD-V) [14], Dynamic Source Routing (DSR) [15], and AODV [16]. Protocols such as Greedy Perimeter Coordinator Routing (GPCR) [17] and Greedy Perimeter Stateless Routing (GPSR) [18] are some of the geographic protocols proposed by researchers when taking vehicle properties into consideration. It is important to mention that the 


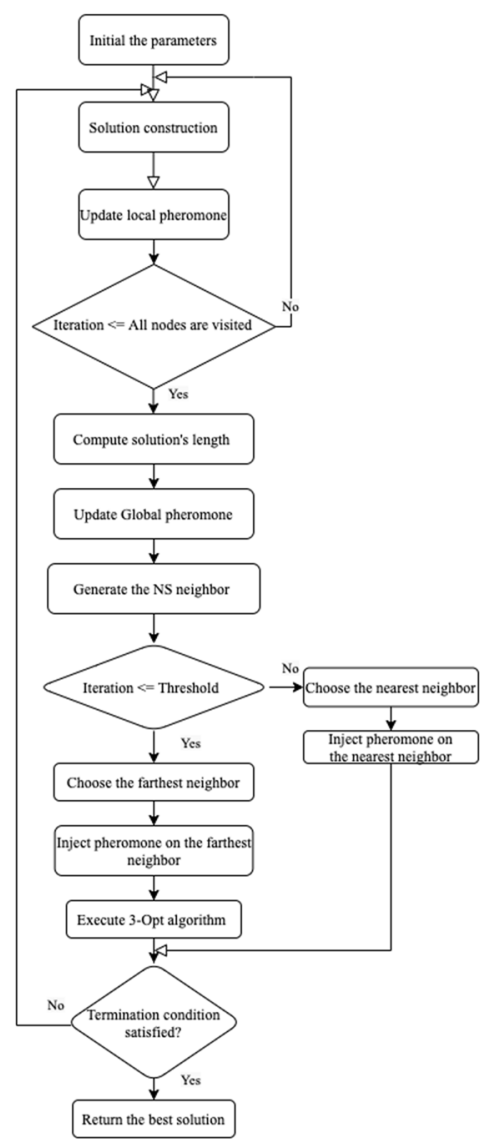

Fig. 1 Dynamic flying ACO flowchart

majority of routing algorithms are only appropriate for solo wireless access technology and limited scale. Therefore, it's very important to evaluate the performance of VANETs routing protocols with other routing algorithms that are designed for VANETs; and not to compare them with the existing routing for MANETs. The overview of VANET routing protocols is shown in Fig. 2 which clearly depicts varieties classification of routing algorithms that is done in this research based on different parameters such as environment, QoS, technique used and protocol type.

The other vast number of protocols for VRP have been introduced $[19,20]$. Providing shortest and best routes by wireless communications is the main objective of all these routing protocols. In this respect, many researches on vehicular routing are conducted based on ACO such as AntRs that best discover a best path among lowest probability of disconnection and shortest route. The outcome of this algorithm proved good adaptability. On the other hand, suitability of this algorithm for dynamic and dense networks, requires more improvement [21]. Combination of proactive and reactive features in order to enhance and preserve the path in VANET, is proposed by G.Caro [22] that is named AntHocNet. This algorithm was inspired from ACO and obtained some improvement on average jitter, delay and ratio of packet delivery but has a low efficiency on routing overhead. A robust ant colony system (ACS) is proposed by Toklu et al. [23] for overcoming the problem of indistinct travel cost. This algorithm utilizes parallel ant colonies and merged the metaheuristic method with a robust optimization method for its purpose. Design of adaptive protocol of VACO (Vehicular routing protocol based on ACO) by Li and Boukhatem [24] was aimed for preserving and creating best paths.

Reduction of communication issues between vehicles is a major challenge for industries and automobile companies such as Car TALK 2000, COMCAR projects, DRIVE, Car Net and Fleet Net [25-30]. Some important parameters that affect the performance reduction of routing protocol are network size and vehicles' speed. The VANET network is a dynamic network, being that there are nodes having inconsistent/random motion leading to nodes frequently experiencing structural deviations. Eventually, this leads to network expiration resulting from the network separation. Therefore, designing an appropriate routing protocol for vehicles in large-scale is a challenging issue. Contribution our study can be explicated as follow:

- Analyzing and measuring the performance of proposed protocols (cDFACO, cR-DFACO, cM-DFACO, cW-DFACO and $\mathrm{CMW}$-DFACO) for VANET environment for supporting $\mathrm{V} 2 \mathrm{I}$ and $\mathrm{V} 2 \mathrm{~V}$ communication.

- Proposing dynamic clustering mechanism by providing Adaptive $\mathrm{CH}$ election based on several parameters (such as vehicular direction, vehicular speed, vehicular position and their distance from each other) in order to form robust communication, less connectivity failure in the network and maximizing the network lifetime. This objective is achieved by compounding of the multiple parameters to elect an optimal $\mathrm{CH}$ among several potential candidates.

- Avoiding network dissemination problems, by enhancing response time and reducing link failure that is achieved by selecting reliable nodes as $\mathrm{CH}$ with low mobility.

- Minimizing the routing cost of admitting request by adapting clustering approach from perspective of bandwidth resource and computing resource consumption for routing its data traffic.

\section{Methodology}

The concentration of this study, is to show different types of possible modification on DFACO and comparison of them with each other and other well-known algorithms to 


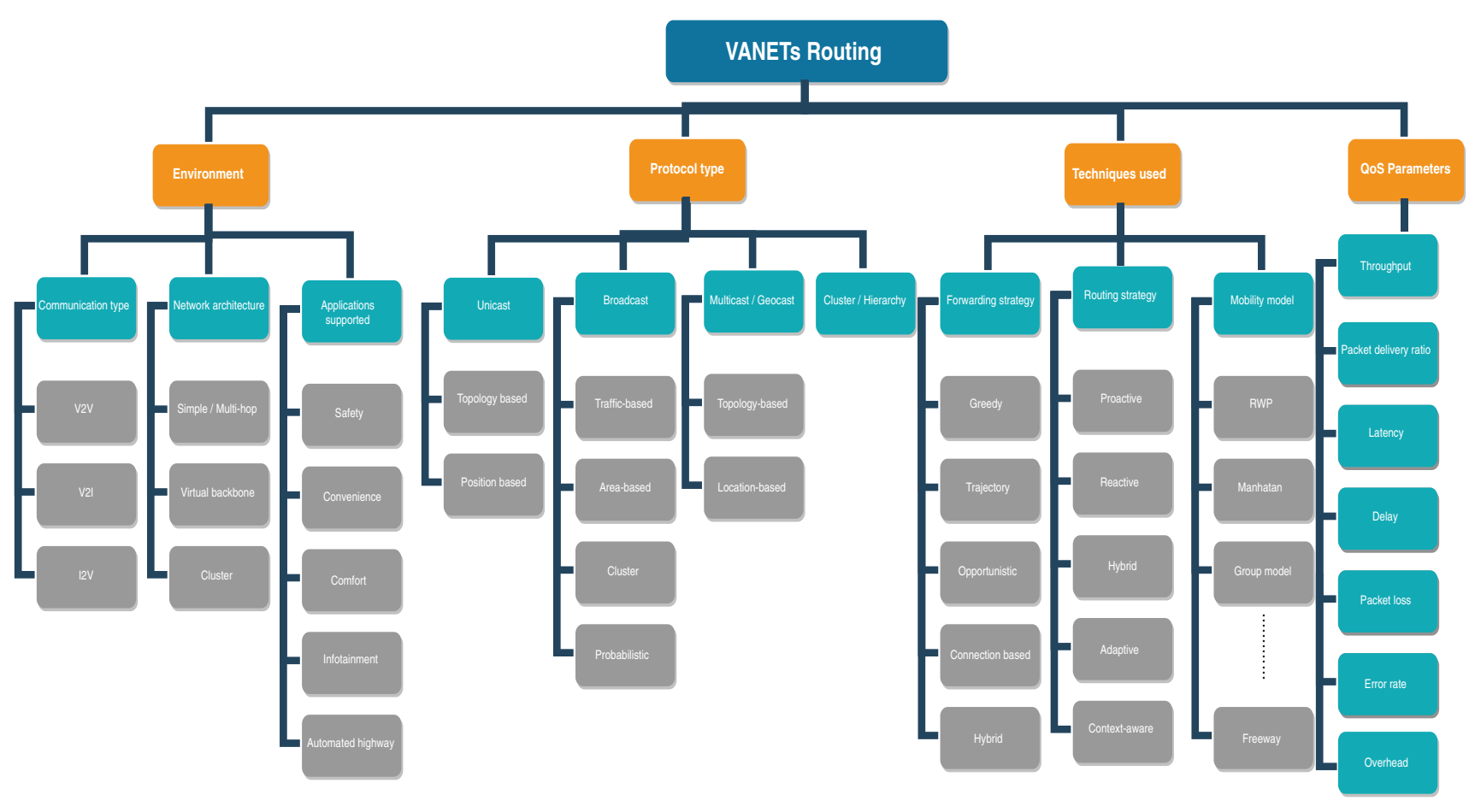

Fig. 2 Taxonomy of VANETs routing protocols

obtain the best solution (which is selecting the best nodes as cluster head) to form more efficient clusters for VANET environment and to fulfill the contribution of this study based on various parameters. In this context, two different stages are considered for performance evaluation of all these proposed algorithms in order to select the best one among them. The phase 1 illustrates the way of clustering the finest feasible paths that are discovered by ants. Moreover, the finest paths/tour indicates the paths with a smaller number of clusters, lower cost/routing cost that cause the shortest path in the entire tour. The presented solution is verified and compared empirically with stateof-the-art baseline techniques like classic ACO [2], DFACO [11] and ACO Based Clustering Algorithm for VANET (CACONET) [31] algorithms in the phase one. CACONET algorithm is the other variation of ACO that is utilized clustering technique and shown better performance than pure ACO in terms of forming a lesser number of clusters in the vehicular network. Considering vehicular transmission range as an only important factor for comparison is the main drawback of this study [31].

Moreover, the best candidate algorithms from the phase 1, are selected to be compared with three wellknown clustering algorithms such as Center Position and Mobility (CPM) [36], Highest-Degree algorithm (HD) [37] and the recent clustering algorithm as Angle-based Clustering Algorithm ACA [38] in terms of network life time in the second phase.

\subsection{Problem mapping}

Pheromone mode is one of the ACO meta-heuristic central components. The pheromone values are normally seen to be related to components of solution. Solutions are probabilistically generated by the pheromone model to the problems being considered by putting them up from the solution components set. The aim of the pheromone update is so that the search is centered in the search space regions that contain solutions with high quality. Particularly, the solution quality reinforcement of solution components is an essential constituent of ACO algorithm. Unreservedly, the system comes to an assumption that good solution components are included in good solutions. Finding out the components that make a good solution can further help in making them even better solutions. Therefore, many researchers get the aide of ACO algorithm for optimizing routing problems to enhance the network performance. In this context, usage of ACO for clustering of nodes in a VANET network is one of the suitable solutions.

An optimal cluster number makes a VANET more stable due to efficient utilization of network resources. In this case, for example, rather than each node doing the job of having the network packets routed to/or within nodes of other clusters, this can be handled by the $\mathrm{CH}$ alone. ACO evolutionary capability aids the proposed algorithm in this study to optimize cluster numbers in the network. In 
algorithms based on ACO, an ant refers to one solution and swarm refers to a group of ants (solutions). So, the aim of this technique is looking out for solutions that are best. These techniques are efficient for discrete and continuous variable problems and are quite efficient. However, compared to other methods their implementation is quite difficult, but also when you compare with other methods. It is computationally inexpensive in situations where there is a need for a comprehensive search to find the optimum solution. What these all mean is that for ad hoc networks, especially VANET. The ACO based techniques are extremely productive for clustering. The first attempt towards achieving efficient clustering in VANET is through ACO. Initially the algorithm finds the $\mathrm{CH}$ and also the neighborhoods.

\subsection{Architecture of CDFACO, cR-DFACO, cM-DFACO, CW-DFACO and CMW-DFACO protocols for VANET}

These proposed algorithms are inspired from DFACO [11] and the recent works based on ACO for Internet of Vehicles (loVs) environment [32] and for Travel Salesman Problems (TSP) [12] which have proved their performance superiorities over many algorithms in the literature. These methods cluster the finest feasible paths that are discovered by ants. Moreover, the finest paths/tour indicates the paths with a smaller number of clusters, lower cost and less execution time that cause the shortest path in the entire tour. The process of pheromone injection by flying ants applies in the same manner for each proposed protocol. Also, the global pheromone updating varies for each proposed method depending on which schemes are selected for comparison but all these algorithms use the same formula to update pheromone locally. Finally, the 3-Opt algorithm combines with the proposed schemes to obtain the best solution quicker. The main structural logic of all these protocols can be seen in Algorithm 1. Also, each of them is explained in more detail as follows.

\subsubsection{CDFACO protocol}

Cluster-based Dynamic Flying Ant (CDFACO) protocol is the modified version of DFACO. The difference of these two algorithms is the applicability of them which CDFACO algorithm is designed for VANET environment; also, is modified in a way that applies clustering technique for finding the shortest path. The overall view of cDFACO scheme is shown in Algorithm 1. The process of clustering vehicles/ nodes are explained on Algorithm 6.

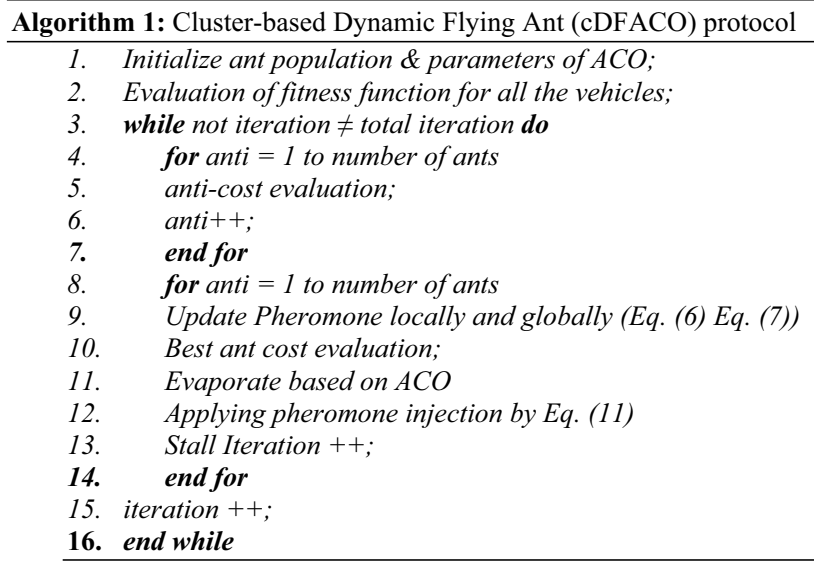

\subsection{2 cM-DFACO protocol}

Cluster-based Modified Dynamic Flying Ant (cM-DFACO) protocol is modification of DFACO algorithm for applicability of that on VANET problem. This algorithm works with a combination of walking and flying ants' functions. Therefore, this method helps the vehicle to be clustered in a more efficient way based on pheromone injection of artificial flying ants to their neighbors. The CM-DFACO protocol applies two phases of modification on CDFACO as dynamic evaporation rate during pheromone update globally and replacing a new pheromone matrix. This novel technique is proposed by our previous study on loV [32] and application of that on TSP [12]. Algorithm 2 illustrates the abstract of this scheme. The execution time of this algorithm is similar to ACO but it prevents being stuck in local optima by balancing the convergence speed. Moreover, discovering the best solution by this algorithm is very accurate and the time complexity of that is less than other proposed algorithms in this study. The mechanism of cM-DFACO involves subsequent stages.

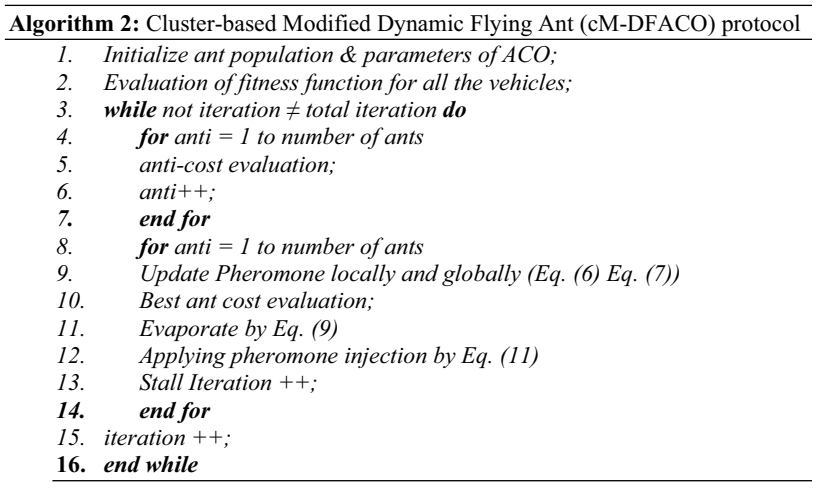


A. Initiating pheromone by using Eq. (2).

$$
\tau_{i j}(\text { iter }=1)=\frac{1}{|M|}
$$

B. The probability of visiting the next vertex by ants is calculated by Eq. (3). By using this method, only the vertex which does not exist in the current tour permit to be added in the tour. The roulette wheel technique is used to execute edge selection which has low computational complexity as $\mathrm{O}(1)$ for preventing algorithms becoming stuck in the local optimum.

$P_{i, j}=\frac{\operatorname{Heu}_{i, j} \cdot \tau_{i, j}}{\sum_{k \in s} \operatorname{Heu}_{i, j} \cdot \tau_{i, j}}$

where Heu and $\tau$ are the heuristic and the pheromone value accordingly. In the progress of transition rule, a novel intelligent method is introduced for electing the first node thru designing a new matrix for pheromone in the search space. For this aim, a column is inserted to the symmetric pheromone matrix of DFACO.

C. Cluster degree: Calculating and normalizing the objective function computes based on Eq. (4) which determines the worth of the obtained solution (ant's tour). Moreover, this formula is used to form dynamic clustering based on ACO, a meta-heuristic algorithm.

$S_{t}=\mathrm{W} 1 \times S_{1}+\mathrm{W} 2 \times S_{2}+\mathrm{W} 3 \times S_{3}$

The W1 $=\mathrm{W} 2=\mathrm{W} 3=0.5$ that shows allocated weights to objective functions $S_{1}, S_{2}$ and $S_{3}$ respectively. $S_{1}$ is the delta difference value between $\mathrm{CH}$ and their $\mathrm{CMs}$. $\mathrm{S}_{2}$ obtains a summation distance of $\mathrm{CH}$ from their $\mathrm{CMs}$ which is based on Euclidean distance [32]. $\mathrm{S}_{3}$ is the distance between nearest neighboring vehicles which can be obtained by Eq. (5)

$$
S_{3}(x, y)=\sqrt{\left(C_{x}-C_{y}\right)^{2}}
$$

where $\mathrm{C}$ is the cost, $\mathrm{x}$ is the cluster head $(\mathrm{CH}) /$ best ants (flying/walking) and $y$ is the cluster members (CM)/ neighboring of flying ants in the tour $t_{i+1}$.

D. Pheromone updating: The nearwhere $C$ is the cost, $\mathrm{x}$ is the cluster head $(\mathrm{CH}) /$ best ants (flying/walking) and $y$ is the cluster members (CM)/ neighboring of flying ants in the tour $t_{i+1}$..est neighboring vehicles receive pheromone from the best ants. Local pheromone updating is calculated based on Eq. (6). In this equation the initial value of pheromone is $\tau_{i j}$ which is obtained from Eq. (2). If the node/vehicle receives pheromone from walking ants, then global update of pheromone applies on the path by using following Eq. (7).

$$
\tau(i, l)=(1-\rho) \tau_{i j}+\rho \tau_{i j}
$$

$\tau(i, l)=(1-\rho)(i, l)+\sum_{k=1}^{m} \Delta \tau^{k}(i, l)$

where $\Delta \tau^{k}(i, l)$ is the number of the pheromone trails in the ant's tour that is secrete by ant $k$ in $\Delta t$ which is specified in following Eq. Where $L_{k}$ is the tour length.

$\Delta \tau^{k}(i, l)=\left(L_{k}\right)^{-1}$

In the Eq. (6), $\rho(0<\rho<1)$ is the rate of evaporation. Also, the value of $\rho$ calculates based on following equation:

$\rho(t)=\tau_{i, l} \times(1-(D E \times c))$

where $C=0.1$ and DE is Dynamic Pheromone Effectiveness which is obtain based on following equation:

$D E= \begin{cases}M I N\left(1, \frac{\left(D C \times \text { Iterration }_{\text {current }}\right)}{\text { Iterations }_{\max }}\right), & \text { if } D C>2 \\ \text { Iterration }_{\text {current }}, & \text { if } D C \leq 2\end{cases}$

where $D C$ is DynamicPheromone_ConvergenceSpeed with the value in the range of [1-10].

E. pheromone injection: $\Delta \tau^{k}(r, s)$ If the node/vehicle receives pheromone from flying ants, then pheromone injection applies on the path by using the following equation.

$\tau_{\left(i+1, x^{\prime}\right)(i+1, L)}(t+1)=\tau_{\left(i+1, x^{\prime}\right)(i, L)}(t)+\left[\frac{\tau_{(i+1, x)\left(i+1, x^{\prime}\right)}(t+1)}{1+\sqrt{\operatorname{dist}_{\text {norm }(\text { Heu })}}}\right]$

where the $\mathrm{CH}$ neighbor's number is determined with $L$ in the rage of $[1, N N]$ and $N N$ is the total number of neighbors for the $\mathrm{CH}$. The dist_norm calculates based on Eq. (12).

$\operatorname{dist}_{\text {norm }}(H e u)=\frac{\operatorname{Heu}_{\left(i+1, x^{\prime}\right)(i+1, L)}}{\sum_{Q=1}^{N N} \operatorname{Heu}_{\left(i+1, x^{\prime}\right)(i+1, Q)}}$

where Heu is the heuristic value in the tour $t_{i+1}$.

\subsection{3 cR-DFACO protocol}

This method is cluster based-Random Dynamic Flaying ACO (CR-DFACO) which inherits all factors of ACO and DFACO but has a difference in updating pheromone. The algorithm of CR-DFACO always has a value of 1 for pheromone and local updating doesn't happen. The global 
updating of this method is based on Eq. (7). This process is shown in Algorithm 3. This protocol is very accrued but the execution time is longer than ACO

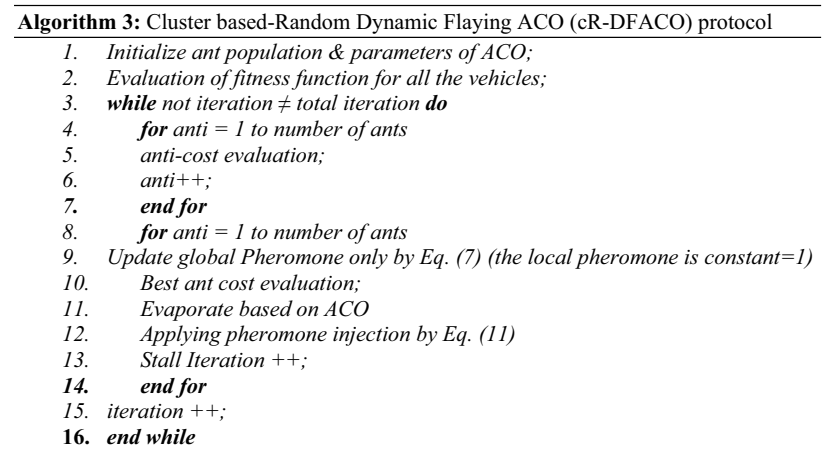

\subsection{4 cW-DFACO protocol}

The Gray Wolf Optimization (GWO) is inspired from social life of wolves and follows four dominant hierarchies such as alpha, beta, gamma and omega which are leaders, subordinates to alpha, subordinates to alpha and beta, and scapegoat role correspondingly [33]. The main aim of using this optimization algorithm can be noted as follow in order to avoid premature convergence of ACO.

- Facilitating retaining the best solutions that obtained in the search space by the social hierarchy of wolves.

- Obtaining higher dimensions as a hyper-sphere around the solutions by using encircling mechanism.

Cluster based-GWO Dynamic Flaying ACO (cW-DFACO) is the other proposed protocol in this research. The objective of DFACO hybridization with Gray Wolf Optimization (GWO) is balancing the path search. In this experimental study we consider three best solutions as alpha, beta and gamma ants as leaders' trials in order to update global pheromone for better exploration in search space [33]. In this aim, a supplementary value of pheromone $\left(w_{i j}\right)$ which obtained from Eq. (13) will be added to the global update pheromone on the ant trail as it is shown in Eq. (14). Algorithm 4 displays the overview of $\mathrm{CW}$-DFACO scheme. where $f_{\alpha}, f_{\beta}$ and $f_{\delta}$ are the fitness values or tour lengths for $\alpha, \beta$ and $\delta$ ants correspondingly. Also, $\rho$ is the Pheromone evaporation rate that has a value of 0.05 .

$$
\varphi(i, l)=\tau(i, l)+w_{i j}
$$

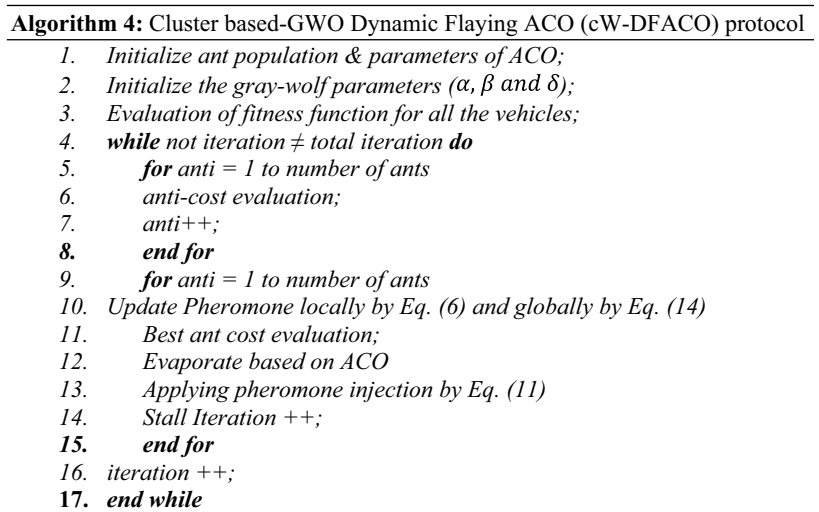

\subsection{5 cMW-DFACO protocol}

Cluster based- modified Dynamic Flaying ACO with GWO (cMW-DFACO) is the last modification that is implemented for this experiment. This method is a hybrid of both $\mathrm{CM}$ DFACO and CW-DFACO algorithms. In this regard, the global updating of pheromone follows the Eq. (14) and the pheromone injection process follows the Eq. (11). In this method $\rho$ is dynamic evaporation rate which obtains from the Eq. (9) that is added to the conventional ACO. Moreover, a new pheromone matrix is replaced by the old pheromone matrix in the process of transition rule. The overall representation of protocol is illustrated in Algorithm 5 .

$W_{i j}= \begin{cases}\left(\frac{3}{f_{\alpha}+f_{\beta}+f_{\delta}}\right) \times(1-\rho) & \text { if } \alpha, \beta \text { or } \delta \text { ants use similar route from } i \text { to } j \text { as ant } K \\ 0 & \text { otherwise }\end{cases}$ 

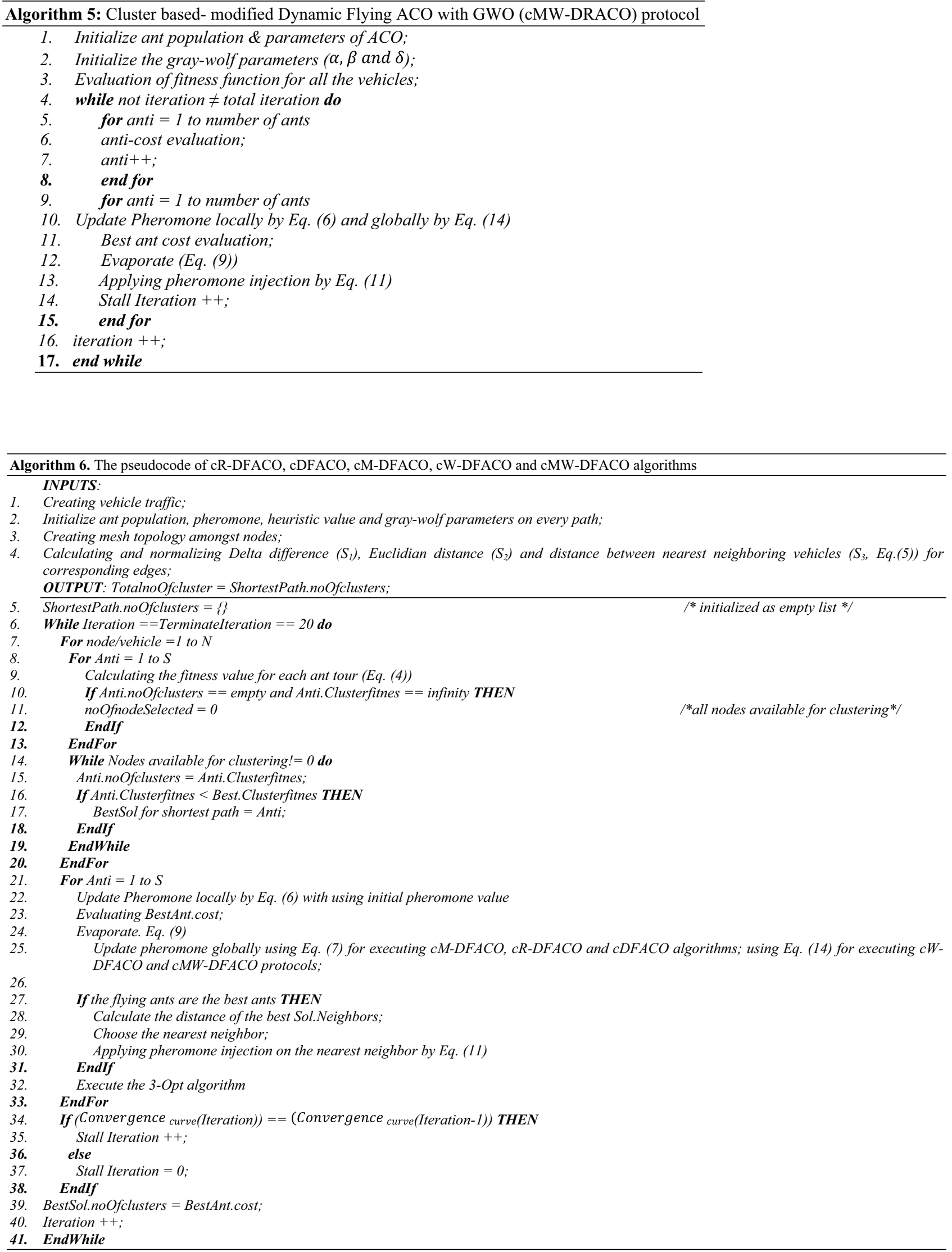

\section{SN Applied Sciences}


Table 1 Simulation setup of phase 1

\begin{tabular}{ll}
\hline Parameter & Value \\
\hline Simulations tool & NS2, SUMO \\
Mobility model & Freeway mobility with 2 intersections \\
MAC layer & WAVE_IEEE 802.11p \\
Interface & Priority queue (50 packets) \\
Queue type & Drop tail \\
Transport layer protocols & UDP/CBR \\
Node movement & Bidirectional \\
Velocity & $22-30 \mathrm{~m} / \mathrm{s}$ uniform \\
Number of RSUs & 4 \\
Number of lanes & 8 \\
Lane width & $50 \mathrm{~m}$ \\
Area & $5 \mathrm{~km} \times 450 \mathrm{~m}$ \\
Number of network nodes & $100,200,300,400$ vehicles \\
Transmission range & $200 \mathrm{~m}$ \\
Packet size & $512 \mathrm{bytes}$ \\
Simulation run & 20 \\
\hline
\end{tabular}

\section{Performance measurement and simulation results}

For this experiment, we implemented 5 types of modifications on DFACO algorithms with considering random positioning of the nodes/vehicles in the highway under SUMO [34] traffic simulator and each simulation is run 20 times. The proposed methods are simulated under NS2 simulation [35]. Two sets of experiments are conducted for evaluating the performance of the proposed protocols. The first set/phase measures solution cost, execution time and average number of clusters that are obtained from each algorithm. Table 1 represents parameters that are utilized for this part of the experiment. All proposed algorithms have constant transmission range (TR) value at $200 \mathrm{~m}$ which is the minimum Dedicated Short-Range Communications (DSRC) coverage for the VANET in highway for obtaining the worst-case performance of proposed protocols in this study. The performance comparison of newly proposed algorithms is done against conventional classic ACO [2], DFACO [11] and CACONET [31] algorithms. Also, Table 2 shows the computational results of this comparison in detail in terms of algorithm running time, average number of cluster and solution cost which are obtained based on the parameters of Table 1. This comparison presented by Figs.3, 4, 5, 6, 7 and 8 in more detail to determine the best performance of routing protocol for the VANETs environment.

The Figs. 3, 4, 5, 6, 7 and 8 illustrate the outcomes of this experimental study that was obtained from phase 1 . Figures 3 and 4 show comparison of all proposed algorithms versus ACO, DFACO and CACONET. This experiment is performed to determine the efficiency of the comparing routing protocols in terms of packet routing cost. In this context, the number of clusters that each algorithm forms should be considered and be compared. The lowest number of cluster formations by routing algorithm is preferred which leads to less routing cost. This research aimed to reduce packet transmission between each cluster which can be achieved by creating efficient clusters (selecting appropriate $\mathrm{CH}$ ) that leads to decrease the number of clusters in the entire network. This objective is obtained by proposing CMDFACO and CR-DFACO schemes. Due to the evolutionary capability of these algorithms larger search spaces can be processed and objective function values can be adjusted dynamically.

The results of Figs. 3 and 4 reveal that by increasing the number of vehicles in a network, the average number of clusters will grow accordingly. Moreover, cR-DFACO is superior to other algorithms in terms of Avg.number of clusters due to $\mathrm{CH}$ selection in this method which is based on the highest heuristic value of nodes. The CMDFACO is the second-best algorithm from this perspective. The highest clusters are formed by DFACO with an average of 24.22 .

Figures 5 and 6 show the comparison of all algorithms with respect to average time for obtaining the best solution. ACO is motivated by the ability of real ant colonies to find shortest paths to a food source. In this research, a rigorous analysis of the execution time of various modified ACO algorithms is conducted for shortest path problems. The obtained results significantly enhance the previous results for shortest paths. The cMW-DFACO and CM-DFACO protocols in every iteration reinforce the best solution found so far. This update strategy is known as best-so-far update; it avoids the problem of stagnation and leads to efficient running times on various problems. Therefore, obtaining the best solution by CMW-DFACO and CMDFACO algorithms takes less time than other algorithms. The CR-DFACO has longest time for discovering the finest solution due to constant and unchanged value of pheromone (which is always 1 ) which effects the speed of algorithm to execute. In this mechanism local updating doesn't perform and the pheromone just updates globally.

All these improved versions of DFACO which are presented and implemented in this study, have in common a stronger exploitation of the best solutions found to direct the ants' search process; they mainly differ in some aspects of the search control. Additionally, cR-DFACO and cMDFACO which are the best performing algorithms for the VANETs routing, improve the solutions generated by the ants using local and global search algorithms. Local search algorithms may enhance the tours formed by the ants. This is typically attained by setting a higher weight to better solutions for the pheromone update and often letting to deposit extra pheromone trails on the arcs of the global 


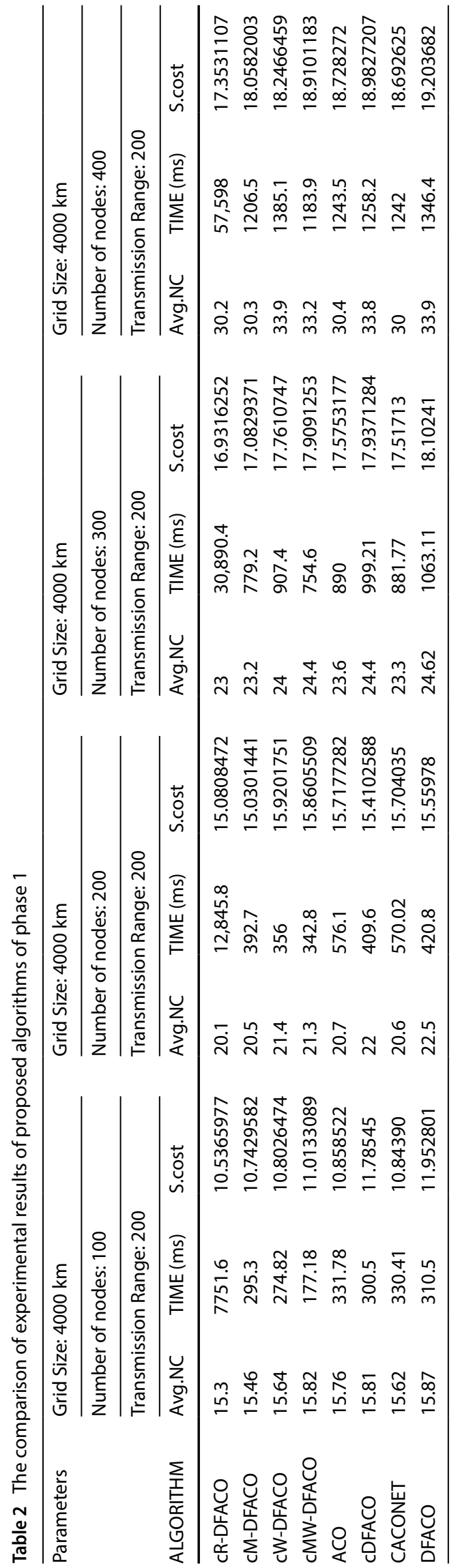

best solution. Figures 7 and 8 show the comparison of all algorithms from the perspective of average solution cost while the number of vehicles increasing from 100 to 400 . The lowest solution cost which shows the accuracy of the routing algorithm belongs to CR-DFACO due to the longest execution time to find the best solution set. Also, the poor solution quality and highest cost belongs to DFACO due to standard local search and standard updating of pheromone locally (based ACO) which affect the quality of ants' search process.

The other objective of this research is to maximize the network lifetime by enhancing the $\mathrm{CH}$ s lifetime which has a direct proportion to network lifetime. Thus, the second set/ phase evaluates cluster lifetime in order to determine robust clustering mechanism by comparing the best proposed algorithms from phase 1 which are CR-DFACO, CMW-DFACO, cM-DFACO with three well-known clustering algorithms such as CPM [36], HD [37] and the recent clustering algorithm as ACA [38]. In this regard, different simulation parameters and performance metrics such as cluster lifetime is considered for evaluating and comparing the stability of presented protocols. Therefore, Table 3 illustrates the simulation parameters for this phase which are taken from NSGA-II approach [39].

In this phase of study, several scenarios are simulated by varying the vehicle density from 20 to 300 vehicles in the whole network. Figure 9 shows the cluster lifetime for all the competing schemes under different vehicle densities. This figure clearly illustrates that the cluster lifetime increases as the number of vehicles increases. It is clear that the proposed schemes in this research attain a considerably longer cluster lifetime than CPM, HD and ACA. Also, CM-DFACO outperforms all the competing schemes according to having the highest cluster lifetime. Moreover, $\mathrm{HD}$ algorithm which selects a node as a cluster head based on the nodes' connectivity has the worst performance in terms of cluster lifetime. The CPM outperforms HD due to cluster formation of the CPM protocol based on the mobility direction. Also, the performance of ACA and CR-DFACO are so close and the cluster lifetime is increased $0.53 \%$ by cR-DFACO algorithm. Also, cM-DFACO increases cluster lifetime by $7.5 \%, 30.6 \%$ and $39.8 \%$ more than ACA, CPM and $\mathrm{HD}$ respectively while considering the high network density in our scenario as 300 vehicles. These results can be explained by the fact that, in CM-DFACO, vehicles with the same direction, closer speed and minimum distance to $\mathrm{CH}$ can form a cluster; therefore, the $\mathrm{CMs}$ will be associated with their $\mathrm{CHs}$ for a longer period of time. The other explanation of highest lifetime by this scheme is several new methodologies and techniques that are performed to the conventional DFACO. 

clusters comparison of all algorithms respect to vehicular density
Fig. 3 Average number of

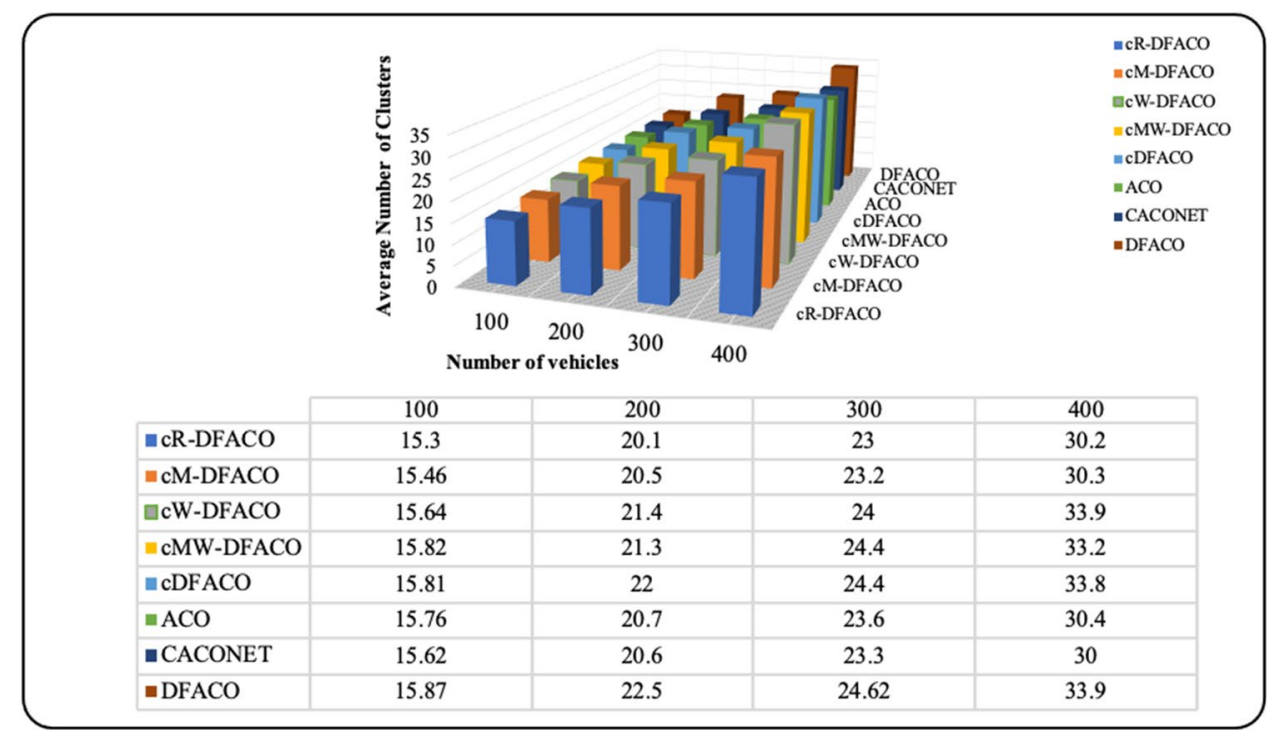

Fig. 4 Comparison of weighted average for number of clusters for all vehicular densities

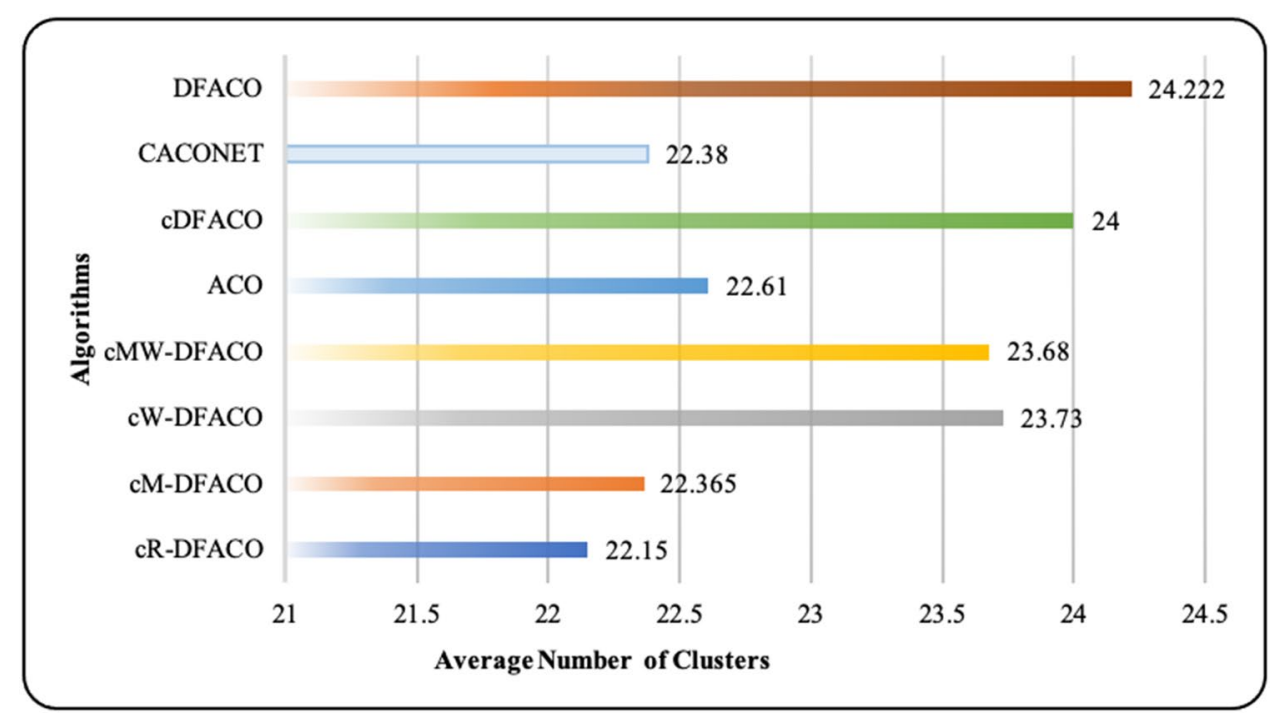

\section{Discussion}

Generally, five important factors affect the behavior of clustering algorithms: (i) Grid size,(ii) Number of nodes, (iii) Routing protocol mechanism, (iv) Vehicular position in the network and (v) Cluster density. Stability of clusters and networks can be attained via minimizing number of clusters. Thus, lesser number of clusters in the network leads to efficient communication and QoS delivery by reduction of routing cost and resources' requirements of the whole network.

In this study, the modification of conventional DFACO is performed in various techniques for balancing exploitation and exploration; and reducing the algorithms' execution time. Moreover, the 3-Opt algorithm is embedded to the proposed algorithms in order to enhance the solution by avoiding local minima which obtains from reconnecting and removing the tour until any further betterment cannot be discovered. Also, all proposed schemes contain a combination of normal and flying ants with the purpose of achieving best solutions with less execution time. In this regard, the achievement is attained in light of two effective and novel concepts. Proposing the dynamic evaporation idea that can be a reason for significantly better performance of all the proposed protocols in this study compared to other algorithms in the literature. This factor balances the convergence procedure by increasing the convergence speed at the start of route detection and slowly reaches to zero dynamically. Enhancing the global search ability is the outcome of this mechanism development in order to optimized clustering in the network. 
Fig. 5 Execution time comparison of all algorithms respect to vehicular density
Fig. 6 Weighted average of execution time for all vehicular densities

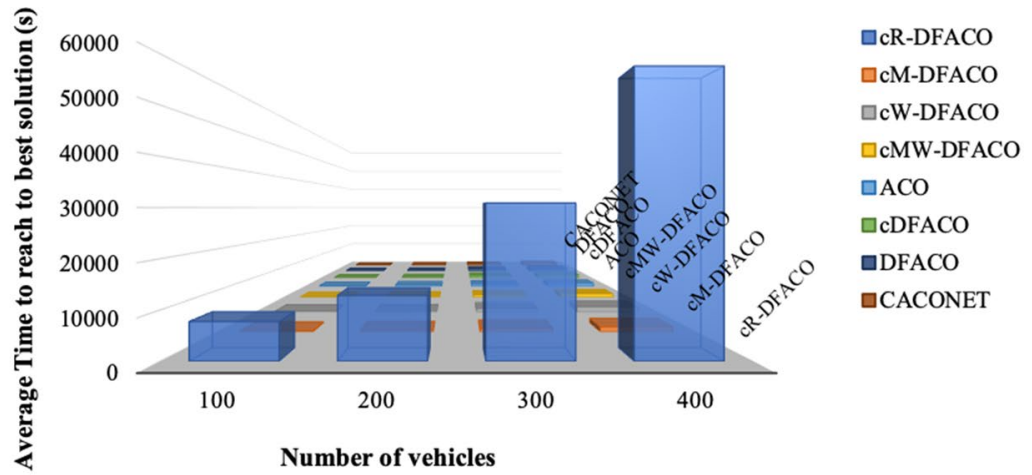

\begin{tabular}{|l|c|c|c|c|}
\cline { 2 - 5 } \multicolumn{1}{c|}{} & 100 & 200 & 300 & 400 \\
\hline$\square \mathrm{cR}-D F A C O$ & 7751.6 & 12845.8 & 30890.4 & 57598 \\
\hline$\square \mathrm{cM}-D F A C O$ & 295.3 & 392.7 & 779.2 & 1206.5 \\
\hline$\square \mathrm{cW}-D F A C O$ & 274.82 & 356 & 907.4 & 1385.1 \\
\hline$\square \mathrm{cMW}-D F A C O$ & 177.18 & 342.8 & 754.6 & 1183.9 \\
\hline$\square$ ACO & 331.78 & 576.1 & 890 & 1243.5 \\
\hline$\square \mathrm{cDFACO}$ & 300.5 & 409.6 & 999.21 & 1258.2 \\
\hline$\square$ DFACO & 310.5 & 420.8 & 1063.11 & 1346.4 \\
\hline$\square$ CACONET & 330.41 & 570.02 & 881.77 & 1242 \\
\hline
\end{tabular}

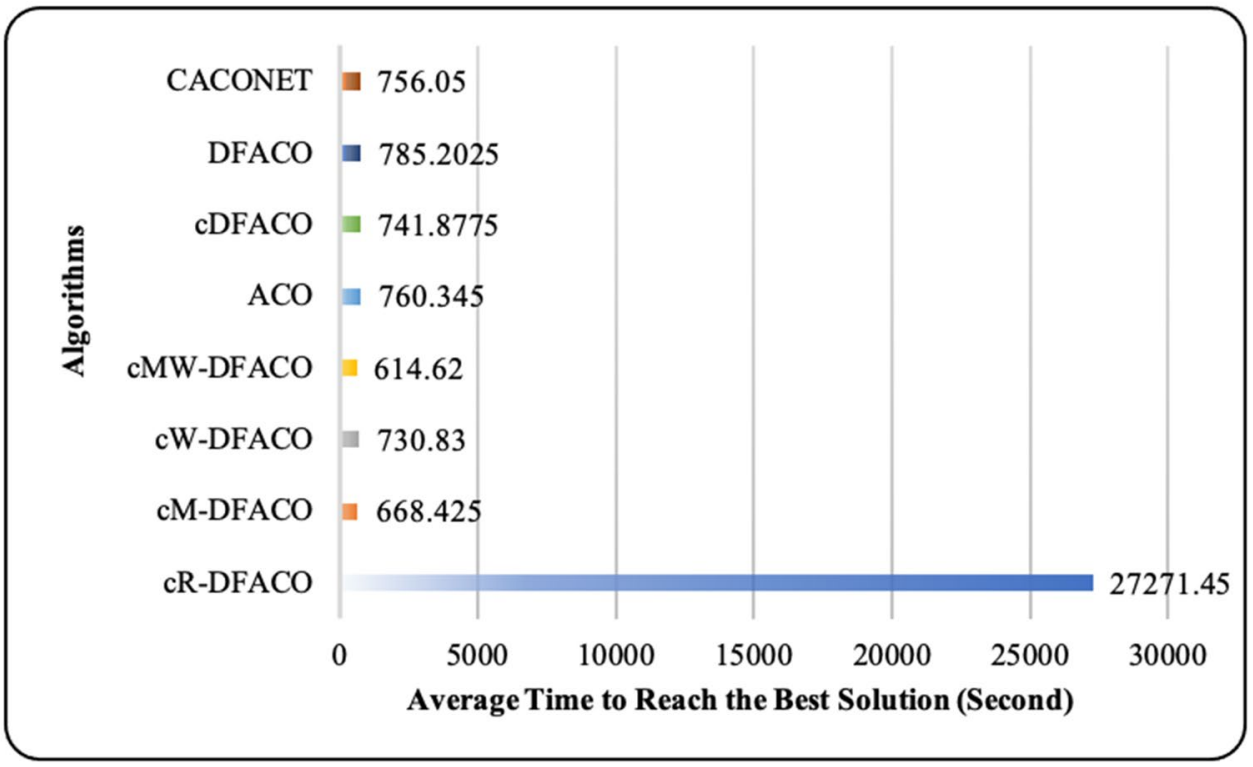

Selecting a suitable vehicle as a $\mathrm{CH}$ is the second efficient idea that is developed in this study. This mechanism, regulating the procedure for updating the pheromone globally which has a straight through effect on the enhancement of cluster stability and increasing the network lifetime.

As it's mentioned earlier, two experiment sets are conducted in this research. The first stage of the experiment concentrates on the different performance metric of competing schemes in order to determine winner protocols in terms of algorithm running time, average number of cluster formation and best solution that obtained by each algorithm. These metrics have direct effect on increasing robustness of vehicular communication and reduction of packet routing cost to cover the whole network. The second stage of the experiment is performed to find the best routing protocol in terms of cluster lifetime which has a direct impact on discovering quality of solution faster. Consequently, the clusters' lifetime and number of $\mathrm{CHs}$ determines the network efficiency.

The goal of proposing DFACO [11] algorithm was achieving better solution with less execution time. Also, CACONET [31] algorithm aimed to form minimum clusters in the network. On the other hand, the proposed framework (cM-DFACO) outperforms the other earliest 
Fig. 7 Average solution cost comparison of all algorithms respect to vehicular density
Fig. 8 Weighted average of solution cost for all vehicular densities
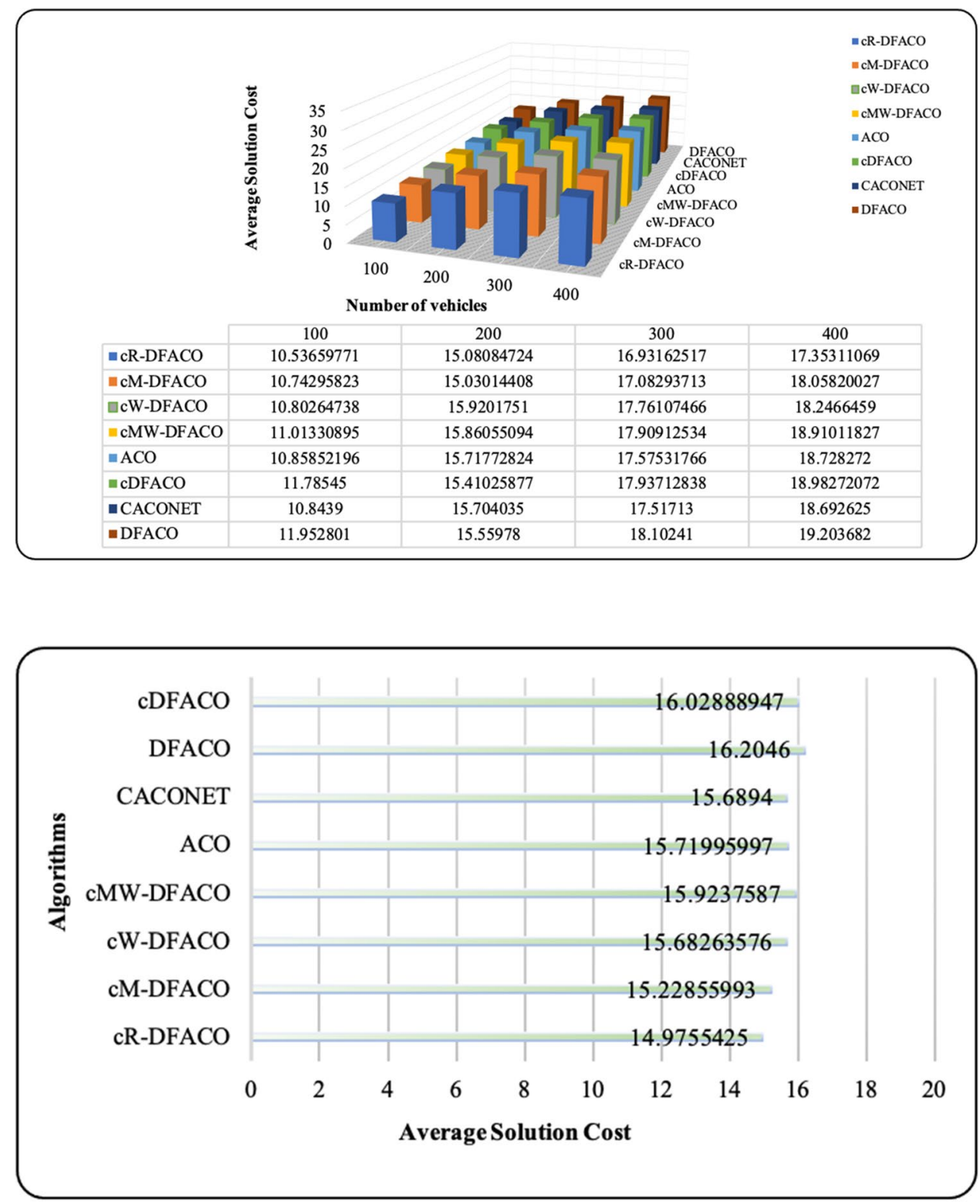

algorithms in the literature in terms of number of clusters, execution time and solution cost respectively. In this regard, the obtained results reveal that the cR-DFACO protocol is very accurate by forming the lowest number of clusters and having lowest solution cost but the execution time of that is much longer than ACO [2]. The longest execution time leads to low cluster lifetime. The DFACO has the worst performance in terms of highest solution cost and highest average number of clusters. Also, the execution time of this algorithm is less than ACO. The solution cost of CACONET is close to the cW-DFACO but has a less performance than to DFACO in terms of Execution time. Also, the number of the clusters that are formed in the network by CACONET is higher than CM-DFACO. The cMW-DFACO algorithm has lowest execution time for discovering the best solution which leads to high cluster lifetime after cM-DFACO. Consequently, the result of the second set of the experiment explicitly illustrates the superiority of cM-DFACO versus all the competing protocols by having the highest cluster lifetime, less execution run time, low solution cost/ routing cost, and low number of clusters in the entire network. Moreover, HD [37] algorithm which selects a node as a cluster head based on the nodes' connectivity has the worst performance in terms of cluster lifetime. The CPM [36] outperforms HD due to cluster formation of the CPM protocol based on the mobility direction. Moreover, cM-DFACO increases cluster lifetime by $7.5 \%, 30.6 \%$ and $39.8 \%$ more than ACA [38], CPM and HD respectively while considering the high network density. Hence, the relevance and added value 
Table 3 Simulation setup of phase 2

\begin{tabular}{ll}
\hline Parameter & Value \\
\hline Simulations tool & NS2, SUMO \\
Mobility model & Freeway mobility \\
Propagation model & Two-Ray-Ground \\
MAC layer & WAVE_IEEE $802.11 \mathrm{p}$ \\
Transport layer protocols & UDP \\
Velocity & $120-150 \mathrm{~km} / \mathrm{h}$ \\
Packet size & 512 bytes \\
Vehicle Distribution & Random \\
Area & $3000 \mathrm{~m} \times 100 \mathrm{~m}$ \\
Traffic Flow & $20,40,80,120,160$, \\
& $200,240,280,300$ \\
Transmission range & $310 \mathrm{~m}$ \\
Simulation time & $80 \mathrm{~s}$ \\
\hline
\end{tabular}

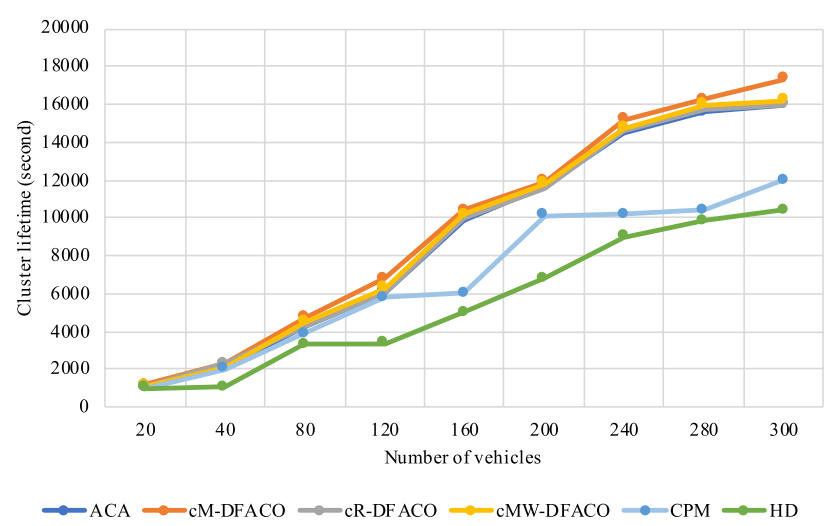

Fig. 9 Cluster lifetime under various traffic densities

of this study is, enhancement of the network efficiency for the VANTEs in light of network stability and minimum routing cost which is applicable by applying cM-DFACO algorithm on the highway for $\mathrm{V} 2 \mathrm{R}$ and $\mathrm{V} 2 \mathrm{~V}$ scenarios while effective communication among all nodes in the VANETs environment is important issue.

\section{Conclusion}

Unsteady network topology and frequent disconnection issues of VANETs motivated this research to propose five unique protocols based-dynamic clustering schemes on DFACO algorithm in order to have minimum number of clusters, high accuracy, minimum time and solution cost. This study contributes to VANETs routing quality using nature-inspired optimization approaches by offering a historic evolution. The implementation of this approach was to find the minimum cost/shortest path for each cluster. Moreover, the applied enhancement on DFACO leads to efficient clustering due to effective strategies for $\mathrm{CH}$ selection by regulating the procedure for updating the pheromone and tuning the evaporation rate that has a major role in ACO. The process of pheromone injection by flying ants applies for each proposed protocol. Also, the global pheromone updating varies for each proposed method depending on which technique is selected but all these algorithms using the same formula update pheromone locally. In this experimental research, proposed protocols are implemented and their performance is analyzed under NS2 and SUMO simulations. The obtained results reveal that the CR-DFACO protocol is very accurate by forming the lowest number of clusters and having lowest solution cost but the execution time of that is much longer than ACO. The CM-DFACO protocol achieved the best result among the other protocols in the literature in terms of highest cluster lifetime, less execution run time, low solution cost and low number of clusters in the entire network. The future work can be conducted based on hybridization of these algorithms with genetic algorithms and evaluate them on the basis of space and time complexity.

Availability of data and material Data are confidential.

Code availability Software application and codes are confidential.

Declarations

Conflicts of interest The author declares that there is no conflict of interest.

Open Access This article is licensed under a Creative Commons Attribution 4.0 International License, which permits use, sharing, adaptation, distribution and reproduction in any medium or format, as long as you give appropriate credit to the original author(s) and the source, provide a link to the Creative Commons licence, and indicate if changes were made. The images or other third party material in this article are included in the article's Creative Commons licence, unless indicated otherwise in a credit line to the material. If material is not included in the article's Creative Commons licence and your intended use is not permitted by statutory regulation or exceeds the permitted use, you will need to obtain permission directly from the copyright holder. To view a copy of this licence, visit http://creativecommons. org/licenses/by/4.0/.

\section{References}

1. Thilak KD, Amuthan A (2017) Survey on improved Artificial bee colony algorithm for balancing exploration and exploitation. Int J Innov Works Eng Technol 3(2):192-200

2. Dorigo M (2007) Ant colony optimization. Scholarpedia 2(3):14-61

3. Sadollah A, Bahreininejad A, Eskandar H, Hamdi M (2013) Mine blast algorithm: A new population-based algorithm for solving constrained engineering optimization problems. Appl Soft Comput 13(5):2592-2612 
4. Sadollah A, Eskandar H, Bahreininejad A, Kim JH (2015) Water cycle algorithm with evaporation rate for solving constrained and unconstrained optimization problems. Appl Soft Comput 30:58-71

5. Mirjalili S, Gandomi AH, Mirjalili SZ, Saremi S, Faris H, Mirjalili SM (2017) Salp swarm algorithm: A bio-inspired optimizer for engineering design problems. Adv Eng Softw 114:163-191

6. Hamza F, Abderazek H, Lakhdar S, Ferhat D, Yıldız AR (2018) Optimum design of cam-roller follower mechanism using a new evolutionary algorithm. Int J Adv Manuf Technol 99(5-8):1267-1282

7. Xu H, Qian X, Zhang L (2012) Study of ACO algorithm optimization based on improved tent chaotic mapping. J Inf Comput Sci 9(6):1653-1660

8. Aljanaby A, Ku-Mahamud KR, Norwawi NM (2010) Interacted multiple ant colonies optimization framework: an experimental study of the evaluation and the exploration techniques to control the search stagnation. Int J Adv Comput Technol 2(1):78-85

9. Lee S, An S (2016) Ant Colony Optimization Algorithm using Back-tracing and Diversification Strategy. In: Proceedings of the world congress on engineering, vol 1

10. Dahan F, El Hindi K, Ghoneim A (2017) An adapted ant-inspired algorithm for enhancing Web service composition. Int J Semant Web Info Sys (IJSWIS) 13(4):181-197

11. Dahan F, El Hindi K, Mathkour H, AISalman H (2019) Dynamic flying ant colony optimization (dfaco) for solving the traveling salesman problem. Sensors 19(8):1837

12. Ebadinezhad S (2020) DEACO: Adopting dynamic evaporation strategy to enhance ACO algorithm for the traveling salesman problem. Eng Appl Artif Intell 92:103-649

13. Dantzig GB, Ramser JH (1959) The truck dispatching problem. Manage Sci 6(1):80-91

14. Perkins $C E$, Bhagwat $P$ (1994) Highly dynamic destinationsequenced distance-vector routing (DSDV) for mobile computers. ACM Comput Commun Rev 24(4):234-244

15. Johnson DB, Maltz DA (1996) Dynamic source routing in ad hoc wireless networks. In: Mobile computing, Springer, Boston, MA. pp 153-181

16. Perkins CE, Royer EM (1999) Ad-hoc on-demand distance vector routing. In: Proceedings WMCSA'99. Second IEEE Workshop on Mob. Comput. Syst. \& Appli. pp 90-100

17. Lochert C, Mauve M, Füßler H, Hartenstein H (2005) Geographic routing in city scenarios. ACM SIGMOBILE Mob Comput Commun Rev 9(1):69-72

18. Karp B, Kung HT (2000) August. GPSR: Greedy perimeter stateless routing for wireless networks. In: Proceedings of the 6 th annual international conference on Mobile computing and networking. pp 243-254

19. Kaur ES (2013) Shortest path finding algorithm using ant colony optimization. Int J Eng Res Technol 2(6):317-326

20. Kumar R, Dave M (2011) A comparative study of Various Routing Protocols in VANET. arXiv preprint http://arxiv.org/abs/1108. 2094

21. Lee KC, Lee U, Gerla M (2010) Survey of routing protocols in vehicular ad hoc networks. In: Advances in vehicular ad-hoc networks: Developments and challenges, IGI Global. pp 149-170

22. Di Caro G, Ducatelle F, Gambardella LM (2005) AntHocNet: an adaptive nature-inspired algorithm for routing in mobile ad hoc networks. Eur T Telecommun 16(5):443-455

23. Toklu NE, Gambardella LM, Montemanni R (2014) A multiple ant colony system for a vehicle routing problem with time windows and uncertain travel times. J Traffic Logi Eng 2:52-58. https://doi. org/10.12720/jtle.2.1.52-58

24. Li G, Boukhatem L (2013) Adaptive vehicular routing protocol based on ant colony optimization, In: Proceeding of the tenth ACM int. workshop on Vehicular inter-networking, systems, \& applic. June, pp 95-98

25. Shastri A, Dadhich R, Poonia RC (2011) Performance analysis of on-demand Routing protocols for vehicular ad-hoc Networks. Int J Wirel Mob Netw (IJWMN) 3(4):103-111

26. Dhaka VS, Poonia RC, Raja L (2014) The realistic mobility evaluation of vehicular Ad-Hoc Network for Indian Automotive Networks. Int J Ad hoc Sensor Ubiquitous Comput. 5(2):1

27. Franz W, Hartenstein H, Mauve M (2005) Inter-vehicle-communications based on ad hoc networking principles. The FleetNet Project, Karlsruhe, Germany: Universtatverlag Karlsruhe. 6:16

28. Festag A, Noecker G, Strassberger M, Lübke A, Bochow B, Torrent-Moreno M, Schnaufer S, Eigner R, Catrinescu C, Kunisch J (2008) NoW-network on wheels: Project objectives, technology and achievements. http://citeseerx.ist.psu.edu/viewdoc/summa ry?doi=10.1.1.219.6798

29. Reichardt D, Miglietta M, Moretti L, Morsink P, Schulz W (2002) CarTALK 2000: Safe and comfortable driving based upon intervehicle-communication. In: Intelligent Vehicle Symposium, IEEE. vol 2, pp 545-550

30. Morris R, Jannotti J, Kaashoek F, Li J, Decouto D (2000) CarNet: A scalable ad hoc wireless network system. In: Proceedings of the 9th workshop on ACM SIGOPS European workshop: beyond the PC: new challenges for the operating system. pp 61-65.

31. Aadil F, Bajwa KB, Khan S, Chaudary NM, Akram A (2016) CACONET: Ant colony optimization (ACO) based clustering algorithm for VANET. PLOS ONE 11(5):e0154080

32. Ebadinezhad S, Dereboylu Z, Ever E (2019) Clusteringbased modified ant colony optimizer for internet of vehicles (CACOIOV). Sustainability 11(9):2624

33. Ab Rashid MFF (2017) A hybrid Ant-Wolf Algorithm to optimize assembly sequence planning problem. Assembly Automation. https://www.emerald.com/insight/content/doi/10.1108/AA-112016-143/full/html?

34. Institute of Transportation Systems, SUMO Simulation of Urban Mobility. Available online: http://sumo.dlr.de/ (accessed on 30 April 2019).

35. The Network Simulator NS-2. 2014. Available online: http:// www.isi.edu/nsnam/ns/ (accessed on 30 April 2019).

36. Lo SC, Lin YJ, Gao JS (2013) A multi-head clustering algorithm in vehicular ad hoc networks. Int J Comput Theory Eng 5(2):242

37. Ramalingam A, Subramani S, Perumalsamy K (2002) Associativity based cluster formation and cluster management in ad hoc networks. In: IEEE HiPC, Bangalore, India, pp 1-6

38. Hadded M, Muhlethaler P, Laouiti A, Saidane LA (2017) A novel angle-based clustering algorithm for vehicular ad hoc networks. In: Vehicular Ad-Hoc Networks for Smart Cities, pp 27-38

39. Hadded M, Zagrouba R, Laouiti A, Muhlethaler P, Saidane LA (2015) A multi-objective genetic algorithm-based adaptive weighted clustering protocol in vanet. In: IEEE congress on evolutionary computation (cec). pp 994-1002

Publisher's Note Springer Nature remains neutral with regard to jurisdictional claims in published maps and institutional affiliations. 